\title{
Sonolência diurna e qualidade do sono em estudantes universitórios de fisioterapia
}

\section{Daytime sleepiness and sleep quality in physical therapy students}

\section{Jarly Oliveira Santos Almeida'; Pedro Paulo Simões de Siqueira'; Anna Myrna Jaguaribe de Lima2; Maria do Socorro Brasileiro-Santos ${ }^{3}$; Valdecir Castor Galindo Filho ${ }^{4}$}

${ }^{1}$ Graduado em Fisioterapia - FIR. Recife, PE - Brasil.

2 Professora Doutora do Departamento de Morfologia e Fisiologia Animal - UFRPE. Recife, PE - Brasil.

${ }^{3}$ Professora Doutora do Departamento de Educação Física - UFPB. João Pessoa, PB - Brasil.

${ }^{4}$ Professor Mestre do curso de Fisioterapia - FIR. Recife, PE - Brasil.

\begin{abstract}
Resumo
Objetivo: Traçar um perfil da qualidade do sono e sonolência diurna dos universitários acadêmicos de graduação em Fisioterapia, possibilitando identificar a prevalência dos distúrbios do sono da amostra analisada. Métodos: Foram entrevistados 260 acadêmicos usando-se o Índice de Qualidade do Sono de Pittsburgh (PSQI) e a Escala de Sonolência de Epworth (ESE). Resultados: Observou-se que $48 \%(n=124)$ dos estudantes apresentavam escores do PSQI $\geq 5$. $\mathrm{Na}$ análise da ESE, $28 \%(\mathrm{n}=74)$ dos participantes apresentavam sonolência diurna classificada entre os três níveis. Conclusão: Neste trabalho, demonstrou-se que os estudantes analisados subestimam sua qualidade do sono. Considerando o papel do sono na vida do indivíduo, um nível de sono não adequado afeta diretamente os compromissos da vida acadêmica e da cotidiana. Portanto, é essencial a realização de mais estudos para identificar os mecanismos que alteram a normalidade dessa função.
\end{abstract}

Descritores: Estudantes; Fisioterapia; Sono; Transtornos da sonolência excessiva.

\begin{abstract}
Objectives: To outline a sleep quality and excessive daytime sleepiness profile of physical therapy undergraduate students, in order to identify the sleep disturbances prevalence in the analyzed sample. Methods: A total of 260 physical therapy students were interviewed through Pittsburgh Sleep Quality Index (PSQI) and Epworth Sleepiness Scale (ESS). Results: We observed that 48\% ( $n=124)$ of the interviewed showed PSQI scores $\geq 5$. About the ESS, $28 \%(n=74)$ of the participants exhibited excessive daytime sleepiness classified between the three levels. Conclusion: In this study, it was demonstrated that physical therapy students minimize their sleep quality. Considering the role of the sleep in the subject life, inadequate sleep quality affects the quotidian and academic commitments. Therefore, further studies are needed to identify mechanisms that modify sleep normal function.
\end{abstract}

Key words: Disorders of excessive somnolence; Physical therapy; Sleep; Students. 


\section{Introdução}

Sono é um estado fisiológico complexo, funcional, reversível e cíclico, organizado em fases que se distinguem por traçados eletroencefalográficos específicos, com manifestações comportamentais e fisiológicas caracterizadas por imobilidade relativa e um maior limiar de resposta aos estímulos externos ${ }^{1,2}$. Embora um indivíduo necessite dormir algumas horas a cada 24 horas, não é a quantidade, e sim a qualidade do sono obtido associada à necessidade individual que determinam a normalidade dessa função ${ }^{3-5}$.

A perturbação do ciclo sono-vigília resulta em significativos danos à saúde e ao bem-estar. Biazus et al. ${ }^{6}$ classificam as consequências dessa perturbação como de curto prazo - provocando cansaço e sonolência durante o dia, irritabilidade, alterações repentinas de humor, perda da memória de fatos recentes, comprometimento da criatividade, redução da capacidade de planejar e executar, lentidão do raciocínio, desatenção e dificuldade de concentração - e de longo prazo - acarretando falta de vigor físico, envelhecimento precoce, diminuição do tônus muscular, comprometimento do sistema imunológico, tendência a desenvolver obesidade, diabetes, doenças cardiovasculares e gastrointestinais e perda crônica da memória.

$\mathrm{Na}$ sociedade moderna, os distúrbios do sono, especialmente a sonolência diurna excessiva, são queixas comuns na população em geral. Na população específica de estudantes universitários, a análise da sonolência diurna é importante, pois eles estão sujeitos a alterações do seu padrão de sono, em razão das modificações que o ingresso no curso de graduação confere ao seu estilo de vida. Além disso, vale ressaltar que na maioria das vezes, os universitários apresentam ansiedade pela pressão de obter um bom desempenho nos estudos, de conseguir sobreviver longe dos pais e de alcançar uma boa qualificação profissional, motivo pelo qual complementam sua formação com atividades extracurriculares, tais como cursos, estágios, iniciação científica e monitoria, o que acarreta ainda mais interferências no sono ${ }^{5,7}$.

Em vista das suas consequências e incidência, os distúrbios do ciclo sono-vigília são considerados um problema de saúde pública, e para os estudantes universitários, as irregularidades desse ciclo acarreta numa diminuição do estado de alerta e concentração no desenvolvimento de atividades acadêmicas ${ }^{7}$. Analisaramse em prévios estudos universitários de diversos cursos de graduação; no entanto, os alunos de graduação em Fisioterapia ainda não foram avaliados quanto aos aspectos do sono ${ }^{8,9-11}$. Dessa forma, propõe-se, com este estudo, traçar um perfil da qualidade do sono e sonolência diurna dos acadêmicos de graduação em Fisioterapia da Faculdade Integrada do Recife (Estácio-FIR), possibilitando identificar a prevalência dos distúrbios do sono na amostra analisada.

\section{Materiais e métodos}

Trata-se de um estudo observacional transversal, aprovado pelo Comitê de Ética e Pesquisa local. Para participarem da pesquisa, os voluntários receberam todas as informações relacionadas aos objetivos e metodologia do estudo e assinaram o Termo de Consentimento Livre e Esclarecido.

A seleção dos participantes deste estudo compreendeu os seguintes critérios de inclusão: ser homem ou mulher entre 18 e 30 anos de idade, estar matriculado no curso de Fisioterapia da Faculdade Integrada do Recife (Estácio-FIR) e não possuir doenças cardiorrespiratórias. Como critérios de exclusão, foram excluídos da amostra os indivíduos que se recusaram a participar e/ou tivessem trancado ou cancelado o curso. Foram avaliados 284 acadêmicos, escolhidos de forma aleatória entre os alunos de graduação da Faculdade Integrada do Recife, entre o $2^{\circ}$ e o $10^{\circ}$ período, entrevistados durante os meses de setembro a novembro de 2010.

Como instrumentos de coleta de dados foram utilizados o Índice de Qualidade do Sono 
de Pittsburgh (PSQI) e a Escala de Sonolência de Epworth (ESE), que são autoaplicáveis.

Para avaliar a qualidade do sono da amostra, foi utilizado o PSQI, que apresenta sensibilidade de $89,6 \%$, e especificidade de $86,5 \% 5$. Quando traduzido e validado para o português, o PSQI mantém sua alta sensibilidade $(80 \%)$; no entanto, uma especificidade menor de $68,8 \%{ }^{8}$. Esse questionário é composto por dez questões, que são agrupadas em sete componentes, cada qual pontuado em uma escala de 0 a 3, sendo eles: a qualidade subjetiva do sono; a latência do sono; a duração do sono; a eficiência habitual do sono; os distúrbios do sono; o uso de medicações para o sono; e a disfunção diurna ${ }^{5,9}$. Os escores dos sete componentes são somados para conferir uma pontuação global do PSQI, a qual varia de 0 a 21. Pontuações superiores a cinco indicam uma qualidade ruim do sono ${ }^{4}$.

Para a avaliação da sonolência diurna excessiva (SDE), utilizou-se a ESE. Estudos clínicos com polissonografia, padrão-ouro para o diagnóstico dos distúrbios do sono, demonstraram que medidas na escala de Epworth acima de dez estão associadas a distúrbios do sono $^{10}$. Essa escala apresenta instruções para pontuação das situações indagadas, sendo a pontuação indicada pelo estudante somada e analisada. Resultados entre 0 e 10 pontos indicam ausência de sonolência; entre 10 e 16 pontos, sonolência leve; entre 16 e 20 pontos, moderada; e entre 20 e 24 pontos, severa ${ }^{5}$.

Para análise dos dados, foi utilizada estatística descritiva por meio do software SPSS, versão 15.0, tendo os resultados sido apresentados como média \pm desvio-padrão e ilustrados por figuras e tabelas.

\section{Resultodos}

Foram avaliados 284 acadêmicos, escolhidos de forma aleatória entre os alunos de graduação da Faculdade Integrada do Recife (EstácioFIR). Desse total, 24 foram excluídos: 6 por estar fora da faixa de idade preestabelecida, 16 por não cursarem a graduação em Fisioterapia e 2 por não responderem os instrumentos completamente. Participaram da pesquisa 260 acadêmicos entre o $2^{\circ}$ e o $10^{\circ}$ semestre do curso de Fisioterapia.

Dos 260 entrevistados que completaram o estudo, 42 (16.15\%) eram homens; 218 (83,85\%) mulheres. Seus dados antropométricos estão apresentados na Tabela 1.

Tabela 1: Índice de qualidade do sono de Pittsburgh em acadêmicos do curso de graduaçõo em Fisioterapia

\begin{tabular}{cccc}
\hline Variáveis & $\begin{array}{c}\text { Homens } \\
(\mathrm{n}=42)\end{array}$ & $\begin{array}{c}\text { Mulheres } \\
(\mathrm{n}=218)\end{array}$ & $\begin{array}{c}\text { Total } \\
(\mathrm{n}=260)\end{array}$ \\
\hline Peso $(\mathrm{kg})$ & $\begin{array}{c}77,17 \pm \\
14,96\end{array}$ & $\begin{array}{c}59,95 \pm \\
10,33\end{array}$ & $\begin{array}{c}61,89 \pm \\
13,04\end{array}$ \\
\hline Altura $(\mathrm{m})$ & $1,76 \pm 0,07$ & $1,63 \pm 0,06$ & $1,65 \pm 0,08$ \\
\hline IMC & $24,85 \pm 4,1$ & $\begin{array}{c}22,22 \pm \\
3,77\end{array}$ & $\begin{array}{c}22,64 \pm \\
3.94\end{array}$ \\
(kg;m $\left.^{2}\right)$ & & $22,20 \pm 1,99$ & $22,25 \pm 2,01$ \\
\hline Idade & $22,5 \pm 2,13$ & 22,0 \\
\hline (anos) & & & \\
\hline
\end{tabular}

Os dados estão apresentados mostrados como média \pm desvio-padrão. kg: quilogramas; $\mathrm{m}$ : metros; $\mathrm{kg} / \mathrm{m}^{2}$ : kilograma por metro quadrado.

A Figura 1 demonstra a qualidade do sono avaliada por meio do PSQI nos estudantes universitários de Fisioterapia. Nesta figura, podese verificar que $48 \% \quad(n=124)$ apresentam uma qualidade do sono ruim, causada por diferentes etiologias entre os estudantes.

Por meio dos diferentes componentes do PSQI, verificou-se que apenas $20 \%(n=52)$ dos entrevistados referiram percepção de uma má qualidade do sono, $85 \%(n=222)$ dos estudantes dormem em média mais de 6 horas por dia e $89 \%$ ( $n=232)$ possuem uma eficiência habitual do sono maior que $85 \%$, ou seja, apresentam uma boa relação entre o número de horas dormidas e o número de horas em permanência no leito, não necessariamente dormindo.

No que diz respeito ao uso de medicamentos para dormir, observou-se que $7 \%(n=18)$ dos estudantes fizeram uso de medicação para dormir por pelo menos uma vez por semana no mês 


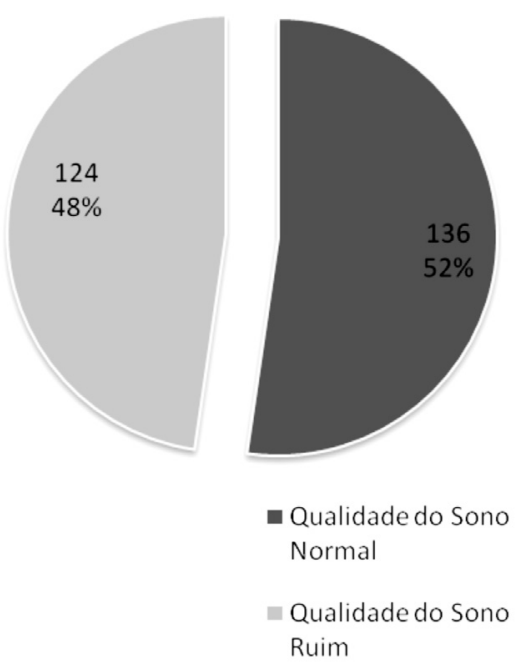

Figura 1: Índice de qualidade do sono de Pittsburgh em acadêmicos do curso de graduação em Fisioterapia. Os dados estão apresentados como valores absolutos e percentual

anterior ao preenchimento dos instrumentos de avaliação. Dentre os distúrbios do sono, destacase que $64,6 \%(n=168)$ dos estudantes apresentavam problemas para dormir por terem sonhos ruins ou pesadelos, $30 \%(n=78)$ referiram dificuldades para dormir por sentirem muito frio ou muito calor menos de uma vez por semana e $46,9 \%$ ( $n=122)$ relataram comprometimento do sono por sentir dores.

Ao avaliar a disfunção durante o dia, que é inerente à sonolência diurna e aos distúrbios diurnos, pode-se verificar que apenas $12 \%$ $(\mathrm{n}=31)$ referiram total disposição para o desenvolvimento das atividades rotineiras (Figura 2).

De acordo com a Figura 2, que representa a SDE dos estudantes de Fisioterapia avaliada pela ESE, observa-se que 28,46\% ( $n=74)$ dos entrevistados apresentavam sonolência diurna classificada entre os três níveis: sonolência leve $(n=66)$, moderada $(n=7)$ e severa $(n=1)$.

\section{Discussão}

A qualidade do sono e seus distúrbios em estudantes têm sido bastante pesquisados nos

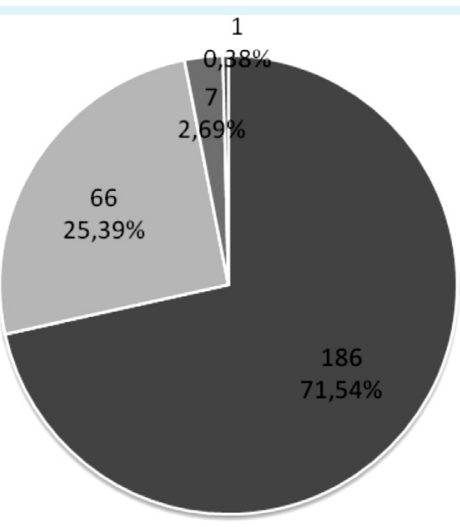

$$
\begin{aligned}
& \text { - Ausência de Sonolência } \\
& \text { - Sonolência Leve } \\
& \text { - Sonolência Moderada } \\
& \text { - Sonolência Severa }
\end{aligned}
$$

Figura 2: Escala de sonolência de Epworth em acadêmicos do curso de graduação em Fisioterapia. Os dados estão apresentados como valores absolutos e percentual

últimos $\operatorname{anos}^{5,711-19}$. Fisiologicamente, o sono é uma necessidade primordial para uma vida saudável, pois ele permite uma restauração física do organismo, preparando-o para as atividades acadêmicas, sociais, dentre outras ${ }^{4}$.

Os questionários utilizados neste estudo

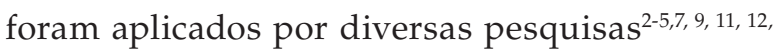
14-16, 20-25, demonstrando a boa aplicabilidade de tais ferramentas para avaliar subjetivamente as informações de caráter qualitativo e quantitativo do sono e sonolência diurna excessiva. Esses instrumentos são acessíveis, simples, de fácil entendimento e preenchimento rápido, ao contrário da polissonografia, que é o padrão ouro para avaliação da qualidade do sono e seus distúrbios, necessitando de um espaço físico adequado, recursos humanos treinados, o que exige um investimento financeiro elevado, restringindo sua acessibilidade a alguns centros clínicos do país ${ }^{26}$.

A partir dos resultados obtidos durante a realização desta pesquisa, verificou-se que a maioria dos estudantes entrevistados (52\%) foram classificados com padrão de normalidade na qualidade do sono. Esse dado corrobora os achados de Trindade-Filho et al. ${ }^{23}$ que 
observaram índices semelhantes de qualidade do sono normal na população adulta da cidade de Maceió-AL. Da mesma forma, Furlani e Ceolim 7 , avaliando estudantes de graduação em enfermagem, demonstraram que 52,3\% dos participantes em período de aula foram classificados com o padrão de sono dentro da normalidade. Em outro estudo, Cardoso et al. ${ }^{5}$ também afirmaram que $85,1 \%$ dos estudantes de graduação e residentes do curso de medicina obtiveram padrão similar.

Ainda, Preišegolavičiūtè et al. $^{12}$, ao avaliarem uma amostra de jovens universitários, em estudo conduzido fora do Brasil, encontraram índices maiores de má qualidade do sono $(59,4 \%)$. Esse achado pode ser explicado devido a diferente adaptação cultural do instrumento de avaliação durante sua tradução e validação com a versão brasileira.

Embora o PSQI apresente uma correlação satisfatória com a polissonografia, os elevados valores encontrados nos diversos estudos podem ser atribuídos ao fato de o questionário ser de caráter subjetivo. Portanto, a utilização da polissonografia nessa mesma amostra poderia apresentar resultados diferentes em virtude da acurácia do método empregado. Neste estudo, considerando os sete componentes avaliados por meio do PSQI, observou-se que os itens que mais se destacaram foram: qualidade subjetiva do sono, duração do sono e uso de medicamentos para dormir.

As queixas de fadiga, sonolência diurna, despertar durante a noite e alterações cognitivas são pouco valorizadas pelos próprios estudantes que tendem a atribuir a desgastes físicos e emocionais. Os achados nesta pesquisa evidenciaram que os estudantes subestimam sua qualidade do sono, uma vez que, embora apenas $20 \%(n=52)$ dos entrevistados tenham referido percepção de uma qualidade do sono ruim, os escores obtidos pelo conjunto das informações de todos os componentes do PSQI, apresentaram valores indicativos dessa condição em $48 \%$ $(n=124)$ dos alunos. Esses resultados também foram relatados na literatura ${ }^{7}$, em que $20,5 \%$ dos estudantes referiram percepção de má qualidade do sono, enquanto que $47,7 \%$ deles apresentavam pontuação indicativa para essa condição.

Quanto à duração do sono, a média de horas dormidas por noite nos estudantes analisados $(6,97 \mathrm{~h})$ foi superior às relatadas em diversos estudos realizados nos estudantes da área de saúde ${ }^{5}$ 7, 12, 16. Na literatura, há algum consenso quanto ao número normal de horas dormidas por noite em adultos jovens, que é de 7 horas ${ }^{3,4}$; porém, vale ressaltar que o número de horas necessárias para proporcionar um sono reparador e de qualidade varia de um indivíduo para o outro. Ainda, uma das dificuldades que surgem na determinação desse tempo está, em grande parte, associada ao próprio estilo de vida e às exigências acadêmicas, o que de acordo com Nércio ${ }^{3}$, muitas vezes, as pessoas dormem não o número de horas que precisam, mas sim o que é possível, sendo assim, geralmente, inferior ao necessário.

Indivíduos submetidos a poucas horas de sono, além de alterações hormonais, sofrem diminuição do metabolismo ${ }^{4}$. Para Berglund et al. ${ }^{27}$, ao investigar 6.599 adultos saudáveis durante um período de dez anos, concluíram que a privação do sono pode aumentar o risco para o desenvolvimento da diabetes mellitus. Entretanto, esses dados não são passíveis de comparação com os resultados deste estudo, pois não foi verificado na amostra analisada o percentual de indivíduos diabéticos.

$\mathrm{O}$ uso de medicamentos para induzir o sono foi relatado por $7 \%(n=18)$ dos estudantes analisados, mostrando-se inferior aos achados de Cardoso et al. ${ }^{5}$ que ao avaliarem estudantes e residentes de medicina encontrou que $8,7 \%$ desses indivíduos fazem uso de medicamentos. Preišegolavičiūtè et al. ${ }^{12}$ encontraram valores ainda menores ao avaliarem estudantes universitários da Lituânia dos cursos de Medicina, Direito e Economia, no qual 5.9\% fazem uso de medicamentos para dormir. Esses dados tornam-se preocupantes, pois nem sempre o uso dessas substâncias tem indicação e acompanhamento médico, podendo provocar mais problemas do que benefícios. Ressalta-se que o uso de 
medicamentos por parte dos estudantes analisados torna-se uma variável de confusão nos achados, pois sua utilização pode induzir uma falsa qualidade do sono referida pelos estudantes avaliados, podendo ser apontado como uma das limitações do estudo.

Somando-se a isso, a SDE é um distúrbio que interfere tanto na qualidade de vida quanto no desempenho cognitivo, atencional, profissional e no relacionamento social, aumentando os riscos de acidentes de trabalho e de trânsito $^{28-30}$. Connor et al. ${ }^{28}$ ressaltam em seu estudo que a proporção de acidentes de trânsito que podem estar relacionados com a SDE nos Estados Unidos é entre $1 \%$ a 3\%; e na Austrália, de 33\%.

Nos achados nesta pesquisa, mostramos que 28,46\% ( $n=74)$ dos estudantes analisados apresentavam algum nível de sonolência diurna. Essa prevalência é inferior à encontrada em estudantes de medicina brasileiros (51,5\%); e nos chilenos (93,2\%), similar aquela encontrada na população em geral $(11,6 \% \text { a } 36 \%)^{5}$, e superior à prevalência observada nos estudantes de Taiwan $(14,4 \%)^{16}$. Salienta-se que a ESE deve ser utilizada para triagem dos distúrbios e indicação para a polissonografia, contribuindo para avaliar qualitativamente e não quantitativamente o grau desse distúrbio; portanto, não deve substituir a polissonografia.

Dessa forma, considerando-se o papel do sono na vida do indivíduo e os efeitos prejudiciais da sua falta, um nível de sono não adequado pode interferir diretamente nos compromissos que as atividades acadêmicas e do dia a dia impõem ao estudante e, assim, afetar sua qualidade de vida. Portanto, cabe aos profissionais de saúde identificar os mecanismos que alteram a normalidade dessa função e promover a conscientização sobre a importância da higiene do sono.

\section{Conclusão}

De acordo com os resultados deste estudo, pode-se concluir que a maioria dos estu- dantes universitários do curso de graduação em Fisioterapia apresentam uma qualidade de sono normal e ausência de sonolência diurna. Sugerese ainda que os estudantes analisados subestimam sua qualidade do sono, pois não consideram as influências negativas as quais são expostos.

É importante que outros estudos sejam realizados a fim de analisar os acadêmicos dos cursos de saúde, entre si e em relação a outras áreas do conhecimento, para uma melhor compreensão dos fatores que influenciam a qualidade do sono desses estudantes universitários.

\section{Referências}

1. Geib LTC, Cataldo Neto A, Wainberg R, Nunes ML. Sono e envelhecimento. Revista de Psiquiatria. 2003;25(3):453-65.

2. Ferro CV. Correlação dos distúrbios do sono e parâmetros subjetivos em indivíduos com fibromialgia. [monografia de graduação]. Cascavel: Unioeste; 2005.

3. Nércio TR. Qualidade do sono e hábitos de estudo em jovens institucionalizados [dissertação de mestrado]. Porto/Portugal: Universidade Fernando Pessoa; 2010.

4. Cunha MCB. Qualidade do Sono em diabéticos do tipo 2 [dissertação de mestrado]. Ribeirão Preto: Universidade de São Paulo; 2006.

5. Cardoso HC, Bueno FCC, Mata JC, Alves APR, Jochims I, Filho IHRV, et al. Avaliação da qualidade do sono em estudantes de Medicina. Rev Bras Educ Med. 2009;33(3):349-55.

6. Biazus JF, Cipolat S, Bordin G, Savoldi CMF, Machado LD, Machado EJU, et al. Qualidade do sono em pacientes atendidos na Clínica Escola de Fisioterapia

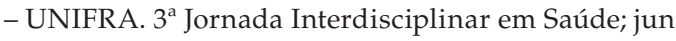
08-11, Santa Maria: Rio Grande do Sul; 2010.

7. Furlani R, Ceolim MF. Padrões de sono de estudantes ingressantes na graduação em Enfermagem. Rev Bras Enferm. 2005;58(3):320-4.

8. Bertolazi AN, Fagondes SC, Perin C, Schonwald SV, John AB, Miozzo ICS, et al. Validation of the Pittsburgh Sleep Quality Index in the brazilian portuguese language. In: Sleep 2008. 22 ${ }^{\text {nd }}$ Annual meeting of the associated professional sleep societies; 2008; Baltimore. Sleep. Westchester: APSS; 2008.v. 31.p. a347. 
9. Fonseca DC, Galdino DAA, Guimarães LHCT, Alves DAG. Avaliação da qualidade do sono e sonolência excessiva diurna em mulheres idosas com incontinência urinária. Rev Neurocienc. 2010;18(3):294-9.

10. Rodrigues RND, Viegas CAA, Silva AAAA, Tavares P. Daytime sleepiness and academic performance in medical students. Arq Neuropsiquiatr. 2002;60(1):6-11.

11. Rosales Mayor E, Egoavil Rojas, MT, La Cruz Dávila CC, Mujica JRC. Somnolencia y calidad de sueño en estudiantes de medicina durante las prácticas hospitalarias y vacaciones. Acta Méd Peruana. 2008;25(4):199-203.

12. Preišegolavičiūtė E, Leskauskas D, Adomaitienè V. Associations of quality of sleep with lifestyle factors and profile of studies among Lithuanian students. Medicina (Kaunas). 2010;46(7):482-9.

13. Mesquita G, Reimão R. Quality of sleep among university students. Arq Neuropsiquiatr. 2010;68(5):720-5

14. Gibson ES, Powler ACP, Thabane L, O'Brien S, Molnar DS, Trajanovic N, et al. Sleepiness" is serious in adolescence: Two surveys of 3235 Canadian students. BMC Public Health. 2006;6:116.

15. Mesquita G, Reimão R. Stress and sleep quality in high school Brazilian adolescents. An Acad Bras Cienc. 2010;82(2):545-51.

16. Kang J-H, Chen S-C. Effects of an irregular bedtime schedule on sleep quality, daytime sleepiness, and fatigue among university students in Taiwan. BMC Public Health 2009,9:248.

17. Suen LKP, Tam WWS, Hon KL. Association of sleep hygiene-related factors and sleep quality among university students in Hong Kong. Hong Kong Med J. 2010;16(3):180-5.

18. Nojomi M, Bandi MFG, Kaffashi S. Sleep Pattern in Medical Students and Residents. Arch Iran Med. 2009;12(6):542-9.

19. Brand S, Hermann B, Muheim F, Beck J. Sleep Patterns, Work, and Strain among Young Students in Hospitality and Tourism. Industrial Health. 2008;46:199-209.

20. Boari L, Cavalcanti CM, Bannwart SRFD, Sofia OB, Dolci JEL. Avaliação da escala de Epworth em pacientes com a Síndrome da apnéia e hipopnéia obstrutiva do sono. Rev Bras Otorrinolaringol. 2004;70(6):752-6.
21. Guimarães KCC. Efeitos dos exercícios orofaríngeos em pacientes com apnéia obstrutiva do sono moderada: estudo controlado e randomizado. [dissertação de doutorado]. São Paulo: Universidade de São Paulo; 2008.

22. Chellapa SL, Araújo JF. Qualidade subjetiva do sono em pacientes com transtorno depressivo. Estudos de Psicologia. 2007;12(3):269-74.

23. Trindade-Filho EM, Carvalho LNA, Gomes EO. Estudo da qualidade do sono na população adulta de Maceió. Revista Neurobiologia. 2010;73(1):93-7.

24. Anderson B, Storfer-Isser A, Taylor HG, Rosen $\mathrm{CL}$, Redline S. Associations of executive function with sleepiness and sleep duration in adolescents. Pediatrics. 2009;123(4):701-7.

25. Barclay, NL, Eley TC, Buysse DJ, Rijsdijk FV, Gregory AM. Genetic and environmental influences on different components of the Pittsburgh sleep quality index and their overlap. Sleep. 2010;33(5):659-68.

26. Bertolazi AN. Tradução, adaptação cultural e validação de dois instrumentos de avaliação do sono: Escala de Sonolência de Epworth e Índice de Qualidade de Sono de Pittsburgh. [dissertação de mestrado]. Porto Alegre: Universidade Federal do Rio Grande do Sul; 2008.

27. Berglund G, Nilsson PM, Rööst M, Engström G, Hedblad B. Incidence of Diabetes in Middle-Aged Men Is Related to Sleep Disturbances. Diabetes Care. 2004;27:2464-9.

28. Connor J, Norton R, Ameratunga S, Robinson E, Civil I, Dunn R, et al. Driver sleepiness and risk of serious injury to car occupants: population based case control study BMJ. 2002;324(7346):1125.

29. Canani SF, Barreto SSM. Sonolência e acidentes automobilísticos. J. Pneumologia. 2001;27(2):94-6.

30. Mulgrew AT, Ryan CF, Fleetham JA, Cheema R, Fox N, Koehoorn M, et al. The impact of obstructive sleep apnea and daytime sleepiness on work limitation. Sleep Med. 2007;9(1):42-53. 